\title{
NNNLO determination of the bottom-quark mass from non-relativistic sum rules
}

\section{Martin Beneke}

Physik Department T31, James-Franck-Straße 1, Technische Universität München, 85748 Garching, Germany

\author{
Andreas Maier ${ }^{* \dagger}$ \\ IPPP, Department of Physics, University of Durham, DH1 3LE, United Kingdom \\ andreas.maier@durham.ac.uk
}

\section{Jan Piclum}

Albert Einstein Center for Fundamental Physics, Institute for Theoretical Physics,

University of Bern, 3012 Bern, Switzerland

Theoretische Physik 1, Naturwissenschaftlich-Technische Fakultät, Universität Siegen,

57068 Siegen, Germany

\section{Thomas Rauh}

Physik Department T31, James-Franck-Straße 1, Technische Universität München,

85748 Garching, Germany

\begin{abstract}
The mass of the bottom quark can be determined with high precision from moments of the pair-production cross section $\sigma\left(e^{+} e^{-} \rightarrow b \bar{b}\right)$ near threshold. We present the first complete NNNLO determination from non-relativistic sum rules, obtaining a bottom-quark mass of $m_{b}^{\mathrm{PS}}(2 \mathrm{GeV})=4.532_{-0.039}^{+0.013} \mathrm{GeV}$ in the potential-subtracted scheme. For the mass in the $\overline{\mathrm{MS}}$ scheme we find $m_{b}^{\overline{\mathrm{MS}}}\left(m_{b}^{\overline{\mathrm{MS}}}\right)=4.203_{-0.034}^{+0.016} \mathrm{GeV}$ using the recently computed four-loop correction to the scheme conversion.
\end{abstract}

12th International Symposium on Radiative Corrections (Radcor 2015) and LoopFest XIV (Radiative Corrections for the LHC and Future Colliders)

15-19 June 2015

UCLA Department of Physics \& Astronomy Los Angeles, CA, USA

\footnotetext{
*Speaker.

${ }^{\dagger}$ Preprint numbers: SI-HEP-2016-01, TUM-HEP-1035/16
} 


\section{Introduction}

A precise knowledge of the bottom-quark mass is of considerable interest not only by itself, but also for a number of phenomenological applications like $B$ meson and Higgs decays.

For a precision determination it is crucial to find a quantity that is both highly sensitive to the mass and well accessible by experiment. This suggests considering the normalised production cross section

$$
R_{b}(s)=\frac{\sigma\left(e^{+} e^{-} \rightarrow b \bar{b}+X\right)}{\sigma\left(e^{+} e^{-} \rightarrow \mu^{+} \mu^{-}\right)}
$$

near threshold. In this region, $R_{b}$ is dominated by non-perturbative effects. Within the sum rule approach [1,2], however, it has been argued that these effects largely cancel out when considering moments

$$
\mathscr{M}_{n}=\int_{0}^{\infty} d s \frac{R_{b}(s)}{s^{n+1}} .
$$

The bottom-quark mass can thus be determined by comparing the weighted integrals over the experimentally measured cross section to the theory prediction for the moments.

For sufficiently large values $n \gtrsim 10$ the moment integral in eq. (1.2) is dominated by the threshold region $\sqrt{s} \sim 2 m_{b}$. In this region, the produced $b \bar{b}$ pair is non-relativistic and the strong interaction leads to bound-state formation and thus a breakdown of conventional perturbation theory. Both of these phenomena are accounted for in the effective theory of potential non-relativistic QCD (PNRQCD) [3], where a simultaneous expansion in $\alpha_{s}$ and the small quark velocity $v \sim 1 / \sqrt{n}$ is performed. Contributions from Coulomb interaction scale as $\alpha_{s} / v$ and are resummed to all orders. More specifically, the power counting up to NNNLO is given by

$$
R_{b} \sim v \sum_{k}\left(\frac{\alpha_{s}}{v}\right)^{k} \times\left\{\begin{array}{ll}
1 & \mathrm{LO} \\
\alpha_{s}, v & \mathrm{NLO} \\
\alpha_{s}^{2}, \alpha_{s} v, v^{2} & \mathrm{NNLO} \\
\alpha_{s}^{3}, \alpha_{s}^{2} v, \alpha_{s} v^{2}, v^{3} & \mathrm{NNNLO}
\end{array} .\right.
$$

To obtain reliable predictions it is necessary that the smallest scale in the theory, given by the kinetic energy $E \sim m_{b} v^{2} \sim m_{b} / n$, remains above the typical scale $\Lambda_{\mathrm{QCD}}$ of non-perturbative physics.

\section{Determination of moments}

In the following we describe the determination of the experimental and theory moments. In both cases we can split the moment integral into a contribution from the narrow bound-state resonances and an integral over the continuum cross section. For the moments $\mathscr{M}_{n}$ with $n \approx 10$ considered in this work the former contribution is dominant.

\subsection{Experimental moments}

Treating the four bound states $\Upsilon(1 S)$ to $\Upsilon(4 S)$ in the narrow-width approximaton we obtain

$$
\mathscr{M}_{n}^{\exp }=9 \pi \sum_{N=1}^{4} \frac{1}{\alpha\left(M_{\Upsilon(N S)}\right)^{2}} \frac{\Gamma_{\Upsilon(N S) \rightarrow l^{+} l^{-}}}{M_{\Upsilon(N S)}^{2 n+1}}+\int_{s_{\mathrm{cont}}}^{\infty} d s \frac{R_{b}(s)}{s^{n+1}} .
$$


To compute the resonance contribution we use the PDG values [4] for the bound-state masses and leptonic widths and the approximation $\alpha\left(M_{\Upsilon(N S)}\right) \sim 1.036 \alpha$ [5] to relate the running QED coupling to the fine structure constant.

The continuum contribution is evaluated by integrating over experimental data [6] corrected for initial-state radiation [7] up to $\sqrt{s}=11.2062 \mathrm{GeV}$ and assuming a flat value of $R_{b}=0.3 \pm 0.2$ for higher energies.

\subsection{Theory moments}

In PNRQCD, the normalised cross section up to NNNLO is given by the master formula [8]

$$
R_{b}=12 \pi e_{b}^{2} \operatorname{Im}\left[\frac{2 N_{c}}{s}\left(c_{v}\left[c_{v}-\frac{E}{m_{b}} \frac{d_{v}}{3}\right] G(E)+\ldots\right)\right]
$$

where $e_{b}$ and $m_{b}$ are the electric charge and pole mass of the bottom quark. The kinetic energy $E$ is related to the center-of-mass energy via $E=\sqrt{s}-2 m_{b}$. The Wilson coefficients $c_{v}$ and $d_{v}$ are obtained by matching the spatial components of the relativistic vector current to non-relativistic currents:

$$
j^{i}=c_{\nu} \psi^{\dagger} \sigma^{i} \chi+\frac{d_{\nu}}{6 m_{b}^{2}} \psi^{\dagger} \sigma^{i} \mathbf{D}^{2} \chi+\ldots
$$

$G(E)$ is the correlator of the non-relativistic current $\psi^{\dagger} \sigma^{i} \chi$. Its poles at $E=E_{N}$ can be interpreted as $\mathrm{S}$-wave bound states; the behaviour near a pole is given by

$$
G(E) \stackrel{E \rightarrow E_{N}}{\longrightarrow} \frac{\left|\psi_{N}(0)\right|^{2}}{E_{N}-E-i \varepsilon},
$$

where $\psi_{N}(0)$ is the wave function at the origin. The theory moments can then be written as

$$
\mathscr{M}_{n}^{\mathrm{th}}=\frac{12 \pi^{2} N_{c} e_{b}^{2}}{m_{b}^{2}} \sum_{N=1}^{\infty} \frac{Z_{N}}{\left(2 m_{b}+E_{N}\right)^{2 n+1}}+\int_{4 m_{b}^{2}}^{\infty} d s \frac{R_{b}(s)}{s^{n+1}}
$$

with the residues

$$
Z_{N}=\frac{4 m_{b}^{2}}{s_{N}} c_{v}\left[c_{v}-\frac{E_{N}}{m_{b}} \frac{d_{v}}{3}\right]\left|\psi_{N}(0)\right|^{2}+\ldots, \quad s_{N}=\left(2 m_{b}+E_{N}\right)^{2} .
$$

According to our power counting (eq. (1.3)) the prefactors $1 / s, 1 / s_{N}$ in eqs. (2.2), (2.6) could be expanded in $E, E_{N} \ll m_{b}$. We find, however, that keeping them in unexpanded form leads to a somewhat better consistency of the mass values extracted from different moments with $n \approx 10$. The difference between the two approaches is within our estimate for the perturbative error.

For the bound-state energies and residues we directly adopt the known NNNLO results [9, $10,11,12,13,14]$. The continuum Green function at NNNLO has mostly been considered in the context of top-pair production $[12,15,16,17]$ and the expressions have to be modified to allow a numerical evaluation in the limit of a vanishing width [18]. In addition to the higher-order QCD corrections we also take into account the leading QED contributions and effects due to a non-zero charm-quark mass up to NNLO [19, 20, 21, 18]. 


\section{Quark mass determination}

To extract the bottom quark mass, we first determine a numerical value for the pole mass via NNNLO conversion [12] from the input mass in the potential-subtracted (PS) scheme [22] and then compute the theory moments from (eq. (2.5)). This corresponds to the PS-shift prescription introduced in [15].

For small renormalisation scales $\mu \lesssim 3 \mathrm{GeV}$ we observe no convergence of the perturbative series, which motivates the choice of $\mu=m_{b}^{\mathrm{PS}}$ as our central scale, and perform a variation within $3 \mathrm{GeV} \leq \mu \leq 10 \mathrm{GeV}$ to estimate the perturbative uncertainty. Even at these comparatively high scales the continuum cross section (cf. figure 1) shows poor behaviour. In particular, there is no clear convergence when going to higher orders, and the NNNLO prediction is incompatible with the fixed-order results for intermediate velocities. Nevertheless, the moments themselves (figure 2) receive only a small contribution from the continuum and appear to be well-behaved as long as $n$ is sufficiently large. Furthermore, the continuum contribution reduces the residual scale dependence of the moments [18]. This suggests that we can indeed extract precise values for the bottom quark mass from moments $\mathscr{M}_{n}$ with $n \approx 10$.
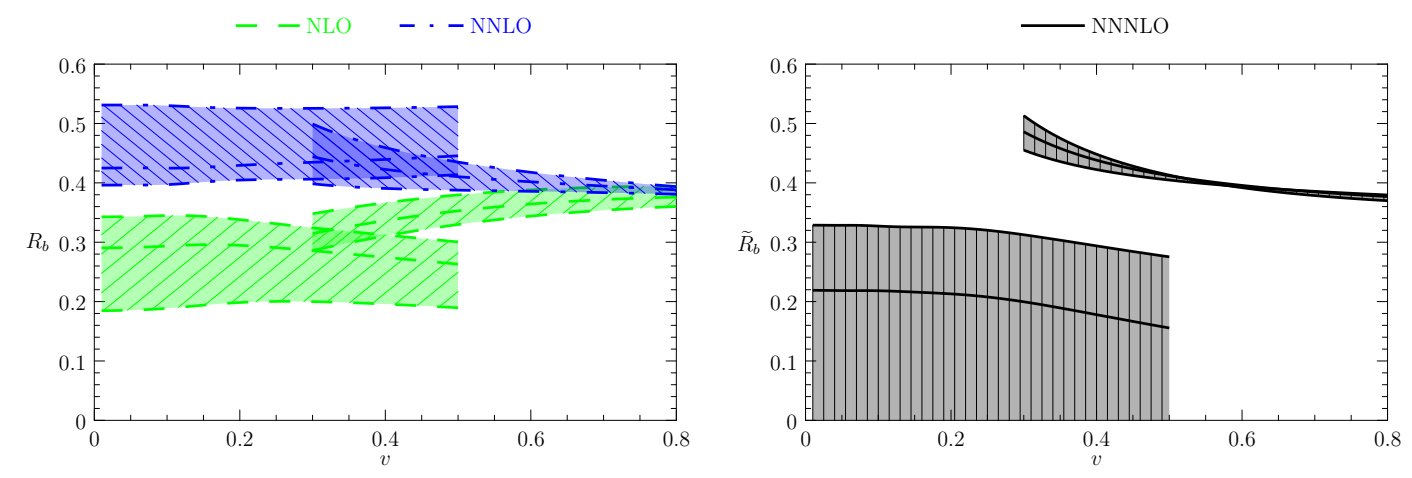

Figure 1: Behaviour of the continuum cross section as a function of $v=\sqrt{E / m_{b}}$ for $m_{b}=5 \mathrm{GeV}$. The curves on the left show the PNRQCD prediction, whereas the curves on the right are a Padé approximation to fixed-order perturbation theory $[23,24]$. The shaded areas arise from varying the renormalisation scale between $3 \mathrm{GeV}$ and $10 \mathrm{GeV}$.

Our main uncertainties for the mass in the PS scheme are due to the perturbative error, estimated as described above, the spread of the mass values extracted from different moments $8 \leq n \leq$ 12 , and the variation of $\alpha_{s}\left(M_{Z}\right)=0.1184 \pm 0.010$. Since we only take into account the first six resonances in the determination of the theory moments (eq. (2.5)), we assign an additional error equal to the difference to the mass value extracted from only four resonances. To estimate the error in the conversion from the PS to the pole scheme we extract $m_{b}^{\mathrm{PS}}$ at some intermediate scale $1 \mathrm{GeV} \leq \mu_{f} \leq 3 \mathrm{GeV}$ and evolve the result to $\mu_{f}=2 \mathrm{GeV}$. For the experimental error we add in quadrature the uncertainties in the $\Upsilon$ masses and leptonic widths, the uncertainty of the available continuum data, and our estimate $0.1 \leq R_{b} \leq 0.5$ for high energies.

In contrast to [21], we find only small corrections due to a non-zero charm-quark mass. We estimate the error from unknown corrections beyond NNLO to be equal to the total charm-mass effect in the bottom-quark mass determination at NNLO. We find very small QED and non-perturbative corrections of less than $1 \mathrm{MeV}$ and neglect the corresponding errors. 


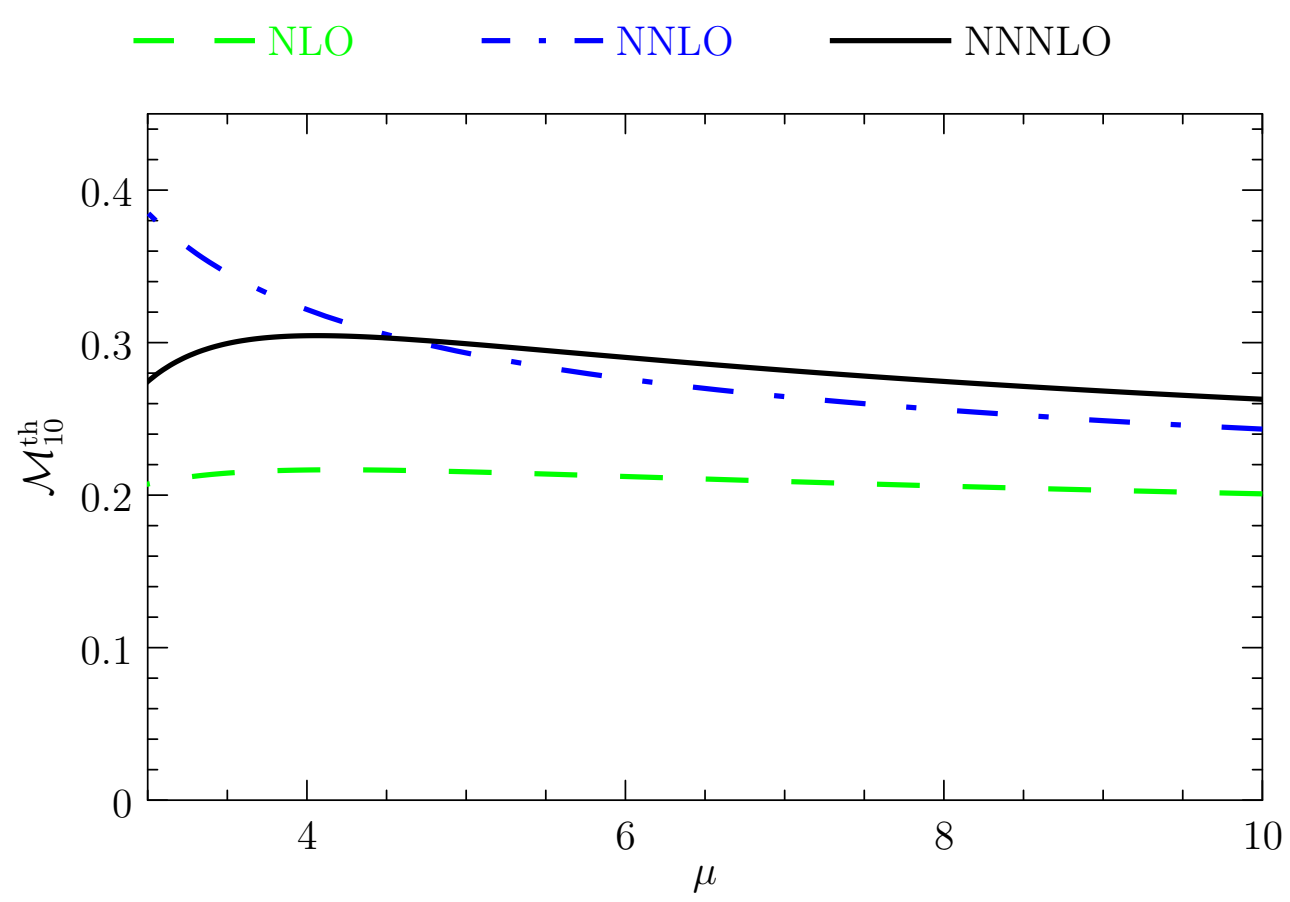

Figure 2: The 10th moment in units of $(10 \mathrm{GeV})^{-20}$ as a function of the renormalisation scale with $m_{b}^{\mathrm{PS}}(2 \mathrm{GeV})=4.5 \mathrm{GeV}$.

As our final result we adopt the bottom-quark mass at a scale of $\mu_{f}=2 \mathrm{GeV}$ determined from the 10th moment. Including the aforementioned uncertainties we obtain

$$
\begin{aligned}
m_{b}^{\mathrm{PS}}(2 \mathrm{GeV})= & {\left[4.532_{-0.035}^{+0.002}(\mu) \pm 0.010\left(\alpha_{s}\right)_{-0}^{+0.003}(\text { res }) \pm 0.001(\text { conv })\right.} \\
& \left. \pm 0.002(\text { charm })_{-0.013}^{+0.007}(n) \pm 0.003(\exp )\right] \mathrm{GeV} \\
= & 4.532_{-0.039}^{+0.013} \mathrm{GeV}
\end{aligned}
$$

From our results for the masses in the PS scheme, we obtain $\overline{\mathrm{MS}}$ masses $m_{b}^{\overline{\mathrm{MS}}}(\bar{\mu})$ using fourloop scheme conversion [25] expressed in terms of the strong coupling constant $\alpha_{s}^{(4)}(\bar{\mu})$ with four active quark flavours. The $\overline{\mathrm{MS}}$ mass scale $\bar{\mu}$ is varied independently between 3 and $10 \mathrm{GeV}$ and the resulting mass is then evolved to $\bar{\mu}=m_{b}^{\overline{\mathrm{MS}}}$. Our results for the $\overline{\mathrm{MS}}$ mass including uncertainties are shown in figure 3 . For the 10th moment at NNNLO we obtain

$$
\begin{aligned}
m_{b}^{\overline{\mathrm{MS}}}\left(m_{b}^{\overline{\mathrm{MS}}}\right)= & {\left[4.203_{-0.031}^{+0.002}(\mu) \pm 0.002\left(\alpha_{s}\right)_{-0}^{+0.003}(\text { res })_{-0.004}^{+0.013}(\text { conv })\right.} \\
& \left. \pm 0.002(\text { charm })_{-0.012}^{+0.006}(n) \pm 0.003(\exp )\right] \mathrm{GeV} \\
= & 4.203_{-0.034}^{+0.016} \mathrm{GeV} .
\end{aligned}
$$

As an alternative to the aforementioned PS-shift prescription, we can use the $\overline{\mathrm{MS}}$ mass $m_{b}(\bar{\mu})$ instead of the PS mass to determine a numerical value for the pole mass used in the computation of the moments. This defines the $\overline{\mathrm{MS}}$-shift prescription. In analogy to the PS-shift treatment, we choose a central renormalisation scale $\mu=m_{b}(\bar{\mu})$ and use $\alpha_{s}^{(4)}(\mu)$ both for the scheme conversion 


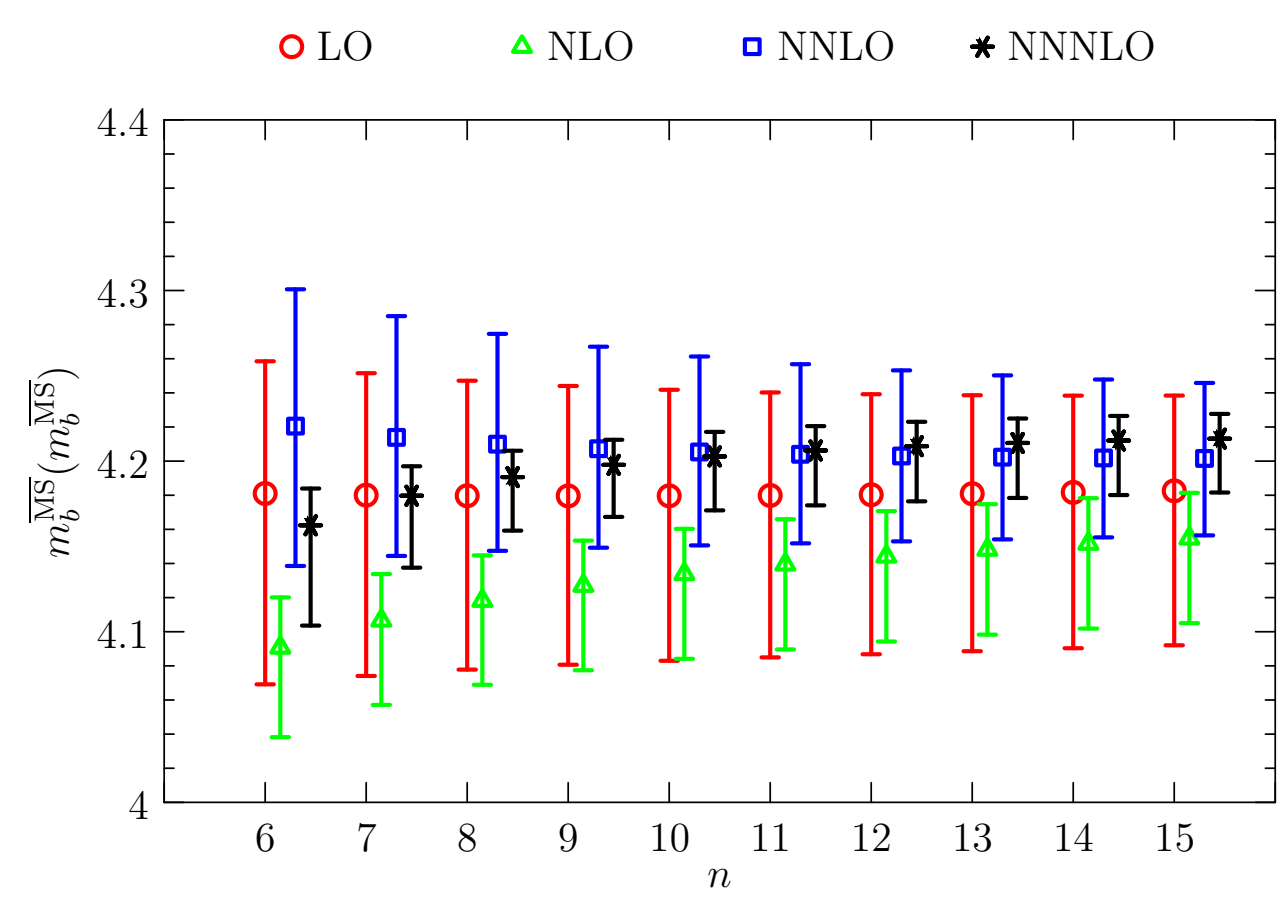

Figure 3: Values for the $\overline{\mathrm{MS}}$ quark mass $m_{b}^{\overline{\mathrm{MS}}}\left(m_{b}^{\overline{\mathrm{MS}}}\right)$ in $\mathrm{GeV}$ extracted from different moments $\mathscr{M}_{n}$ using the PS-shift prescription.

and the calculation of the moments. We arrive at

$$
\begin{aligned}
m_{b}^{\overline{\mathrm{MS}}}\left(m_{b}^{\overline{\mathrm{MS}}}\right)= & {\left[4.204_{-0.019}^{+0.000}(\mu) \pm 0.002\left(\alpha_{s}\right)_{-0}^{+0.003}(\text { res })_{-0.005}^{+0.002}(\text { conv })\right.} \\
& \left. \pm 0.002(\mathrm{charm})_{-0.013}^{+0.007}(n) \pm 0.003(\exp )\right] \mathrm{GeV} \\
= & 4.204_{-0.024}^{+0.008} \mathrm{GeV} .
\end{aligned}
$$

While the central value is in excellent agreement with eq. (3.2), the scale uncertainty in eq. (3.3) is considerably smaller. In fact, it does not cover the central value $m_{b}^{\overline{\mathrm{MS}}}\left(m_{b}^{\overline{\mathrm{MS}}}\right)=4.177 \mathrm{GeV}$ we obtain when expanding the prefactors $1 / s, 1 / s_{N}$ in eqs. (2.2), (2.6) as discussed in section 2.2. Since the preference for the unexpanded prefactors is not based on systematic considerations, as discussed in [18], we quote the estimate eq. (3.2) as our final result.

Compared to [18], our results for the $\overline{\mathrm{MS}}$ mass are shifted upwards by $10 \mathrm{MeV}$ and the uncertainty from the scheme conversion is reduced significantly. These changes are due to the recently calculated value for the four-loop coefficient in the scheme conversion [25], which is smaller by about $8 \%$ compared to the estimate [26] used in [18].

\section{Acknowledgements}

This work has been supported by the DFG Sonderforschungsbereich/Transregio 9 "Computergestützte Theoretische Teilchenphysik", the Gottfried Wilhelm Leibniz programme of the Deutsche Forschungsgemeinschaft (DFG), and the Munich Institute for Astro- and Particle Physics (MIAPP) of the DFG cluster of excellence "Origin and Structure of the Universe". A. M. is supported by a 
European Union COFUND/Durham Junior Research Fellowship under EU grant agreement number 267209.

\section{References}

[1] V. Novikov, L. Okun, M. A. Shifman, A. Vainshtein, M. Voloshin and V. I. Zakharov, Sum rules for charmonium and charmed mesons decay rates in quantum chromodynamics, Phys. Rev. Lett. 38 (1977) 626.

[2] V. Novikov, L. Okun, M. A. Shifman, A. Vainshtein, M. Voloshin and V. I. Zakharov, Charmonium and gluons: Basic experimental facts and theoretical introduction, Phys. Rept. 41 (1978) 1-133.

[3] A. Pineda and J. Soto, Effective field theory for ultrasoft momenta in NRQCD and NRQED, Nucl. Phys. Proc. Suppl. 64 (1998) 428-432, [arXiv:hep-ph/9707481].

[4] Particle Data Group collaboration, J. Beringer et al., Review of particle physics (RPP), Phys. Rev. D86 (2012) 010001.

[5] F. Jegerlehner, Electroweak effective couplings for future precision experiments, Nuovo Cim. C034S1 (2011) 31-40, [arXiv:1107.4683].

[6] BABAR collaboration, B. Aubert et al., Measurement of the $e^{+} e^{-} \rightarrow b \bar{b}$ cross section between $\sqrt{s}=$ 10.54-GeV and 11.20-GeV, Phys. Rev. Lett. 102 (2009) 012001, [arXiv:080 . 4120].

[7] K. Chetyrkin, J. Kühn, A. Maier, P. Maierhöfer, P. Marquard, M. Steinhauser et al., Charm and bottom quark masses: An update, Phys. Rev. D80 (2009) 074010, [arXiv:0 907.2110$].$

[8] M. Beneke, Y. Kiyo and K. Schuller, Third-order correction to top-quark pair production near threshold I. Effective theory set-up and matching coefficients, [arXiv:1312.4791].

[9] B. A. Kniehl and A. A. Penin, Ultrasoft effects in heavy quarkonium physics, Nucl. Phys. B563 (1999) 200-210, [arXiv:hep-ph/9907489].

[10] B. A. Kniehl, A. A. Penin, V. A. Smirnov and M. Steinhauser, Potential NRQCD and heavy quarkonium spectrum at next-to-next-to-next-to-leading order, Nucl. Phys. B635 (2002) 357-383, [arXiv:hep-ph/0203166].

[11] A. Penin, V. A. Smirnov and M. Steinhauser, Heavy quarkonium spectrum and production/annihilation rates to order $\beta_{0}^{3} \alpha_{s}^{3}$, Nucl. Phys. B716 (2005) 303-318, [arXiv:hep-ph/0501042].

[12] M. Beneke, Y. Kiyo and K. Schuller, Third-order Coulomb corrections to the S-wave Green function, energy levels and wave functions at the origin, Nucl. Phys. B714 (2005) 67-90, [arXiv:hep-ph/0501289].

[13] M. Beneke, Y. Kiyo and A. Penin, Ultrasoft contribution to quarkonium production and annihilation, Phys. Lett. B653 (2007) 53-59, [arXiv:0 706 .2733].

[14] P. Marquard, J. H. Piclum, D. Seidel and M. Steinhauser, Three-loop matching of the vector current, Phys. Rev. D89 (2014) 034027, [arXiv:1 401.3004 ].

[15] M. Beneke, Y. Kiyo and K. Schuller, Third-order correction to top-quark pair production near threshold II. Potential contributions, In preparation .

[16] M. Beneke and Y. Kiyo, Ultrasoft contribution to heavy-quark pair production near threshold, Phys. Lett. B668 (2008) 143-147, [arXiv:0 804.4004 ]. 
[17] M. Beneke, Y. Kiyo, P. Marquard, A. Penin, J. Piclum and M. Steinhauser, Next-to-Next-to-Next-to-Leading Order QCD Prediction for the Top Antitop S-Wave Pair Production Cross Section Near Threshold in $e^{+} e^{-}$Annihilation, Phys. Rev. Lett. 115 (2015) 192001, [arXiv:1506.06864].

[18] M. Beneke, A. Maier, J. Piclum and T. Rauh, The bottom-quark mass from non-relativistic sum rules at NNNLO, Nucl. Phys. B891 (2015) 42-72, [arXiv:1411.3132].

[19] M. Melles, Massive fermionic corrections to the heavy quark potential through two loops, Phys. Rev. D58 (1998) 114004, [arXiv:hep-ph/9805216].

[20] M. Melles, The static QCD potential in coordinate space with quark masses through two loops, Phys. Rev. D62 (2000) 074019, [arXiv:hep-ph/ 0001295 ].

[21] A. Hoang, Bottom quark mass from \ mesons: Charm mass effects, [arXiv:hep-ph/0 008102 ].

[22] M. Beneke, A quark mass definition adequate for threshold problems, Phys. Lett. B434 (1998) 115-125, [arXiv:hep-ph/9804241].

[23] A. H. Hoang, V. Mateu and S. Mohammad Zebarjad, Heavy quark vacuum polarization function at $\mathscr{O}\left(\alpha_{s}^{2}\right)$ and $\mathscr{O}\left(\alpha_{s}^{3}\right)$, Nucl. Phys. B813 (2009) 349-369, [arXiv:0807.4173].

[24] Y. Kiyo, A. Maier, P. Maierhöfer and P. Marquard, Reconstruction of heavy quark current correlators at $\mathscr{O}\left(\alpha_{s}^{3}\right)$, Nucl. Phys. B823 (2009) 269-287, [arXiv:0907.2120].

[25] P. Marquard, A. V. Smirnov, V. A. Smirnov and M. Steinhauser, Quark Mass Relations to Four-Loop Order in Perturbative QCD, Phys. Rev. Lett. 114 (2015) 142002, [arXiv:1502 . 01030].

[26] C. Ayala, G. Cvetič and A. Pineda, The bottom quark mass from the $\Upsilon(1 S)$ system at NNNLO, JHEP 1409 (2014) 045, [arXiv:1407.2128]. 